\title{
Moyamoya Syndrome with Primary Antiphospholipid Syndrome
}

\author{
Mohammed Ali Shuja-Ud-Din ${ }^{\mathrm{a}}$ Shefeek Abubacker Ahamed ${ }^{\mathrm{b}}$ \\ Ghassan Baidas $^{\text {a }}$ Mohammad Naeem ${ }^{a}$ \\ Departments of a Medicine and ${ }^{\mathrm{b}} \mathrm{Clinical}$ Radiology, Al-Sabah Hospital, Kuwait
}

\author{
Key Words \\ Moyamoya disease - Antiphospholipid syndrome • \\ Stroke
}

\begin{abstract}
Objectives: To report a rare case of moyamoya syndrome with primary antiphospholipid syndrome (APS). Clinical Presentation and Intervention: A 41-year-old woman was admitted with sudden onset of left-sided hemiparesis. Magnetic resonance imaging and magnetic resonance angiography of the brain showed characteristic features of moyamoya vessels. Laboratory investigations revealed raised levels of anticardiolipin antibody. She made a complete and uneventful recovery with aspirin and intensive physiotherapy over a period of 2 weeks. Conclusion: The association of APS and moyamoya disease may present a dilemma in management. While APS is often considered to be an indication for giving anticoagulation, a trial of antiplatelet agents is preferable to anticoagulation because of the risk of bleeding from the fragile moyamoya vessels.
\end{abstract}

Copyright (C) 2006 S. Karger AG, Basel

\section{Introduction}

Moyamoya disease is a rare, progressive cerebrovascular disorder characterized by the narrowing or occlusion of major vessels to the brain [1]. Moyamoya in Japanese means 'puff of smoke' because of the appearance of the collateral vessels on the cerebral angiogram [1]. Moyamoya syndrome (MMS) refers to cases in which the characteristic angiographic findings are observed in young adults. In North America MMS is most commonly diagnosed in women, and it is associated with use of oral contraceptives and tobacco abuse [2]. Antiphospholipid antibody syndrome (APS) is diagnosed when a thrombotic episode is associated with antiphospholipid antibody detected on two occasions at least 3 months apart [3]. The repeated strokes can lead to severe functional impairment or even death. Hence it is important to recognize these lesions and treat them early on. To the best of our knowledge this is the first case report in the Middle East of MMS with superimposed APS treated solely with antiplatelet agents.

\section{Case Report}

A 41-year-old right-handed Kuwaiti woman was admitted with sudden onset of left-sided hemiparesis. She had a history of smoking, approximately 20 packs of cigarettes a year. She had no history of spontaneous abortion, deep vein thrombosis, transient ischemic attacks, orogenital ulcers or use of contraceptive pills. On physical examination she was fully oriented and alert together with left upper motor facial weakness and a power of grade 3 in both upper and lower limbs. No carotid artery, ocular or temporal bruits or cardiac murmurs were detected. Laboratory tests and electrocardiogram were normal. $\mathrm{T}_{2}$-weighted magnetic resonance images of the brain revealed multiple infarcts involving the right temporal lobe, right internal capsule and the centrum semiovale bilaterally (fig. 1). Contrast-enhanced magnetic resonance angiography (MRA) of the extracranial carotids showed tubular narrowing of the right internal carotid artery (ICA)

\section{KARGER}

Fax +41613061234 E-Mail karger@karger.ch www.karger.com
Dr. Shuja-Ud-Din 

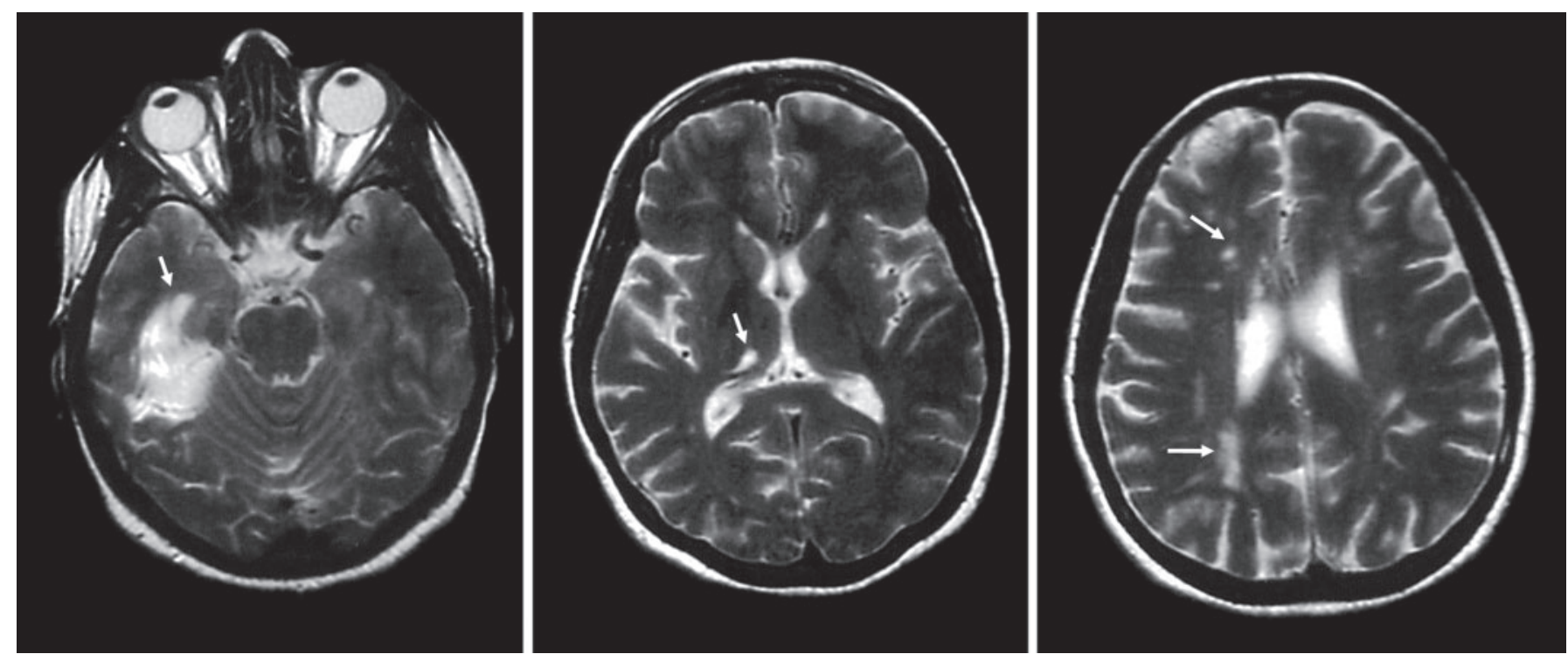

Fig. 1. $T_{2}$-weighted MR images of the brain reveal multiple infarcts of the brain involving the right temporal lobe, right internal capsule and bilateral semiovale (arrows).

but normal left ICA (fig. 2). Three-dimensional time of flight (3DTOF) imaging of the circle of Willis (fig. 3, 4) revealed occlusion of the supraclinoid segment of the right ICA together with significant narrowing of the right middle cerebral artery (MCA) and posterior cerebral artery (PCA) and extensive collateralization arising from the right MCA and PCA giving the hallmark appearance of a 'puff of smoke'. Right and left anterior cerebral arteries, left MCA, PCA and ICA were normal (fig. 3, 4). Based on the cerebrovascular pattern, a radiological diagnosis of moyamoya disease was made.

Screening for a hypercoagulable state includes assays of protein $\mathrm{C}$, protein S, antithrombin III, Factor V Leiden, lupus anticoagulant, anticardiolipin antibodies (aCL), and anti- $\beta_{2}$-glycoprotein 1 antibodies ( $\beta_{2}$ GPI). On admission patient's $\beta_{2}$ GPI were IgA $180 \mathrm{SAU}$ (normal value $20 \mathrm{SAU}$ ) and IgM $27 \mathrm{SMU}$ (normal value $20 \mathrm{SMU}$ ), aCL $45 \mathrm{U} / 1$ (normal value $13.3 \mathrm{U} / 1$ ). Two months later the level of aCL decreased to $23 \mathrm{U} / 1$ and $\beta_{2}$ GPI IgA to 67 SAU. Antinuclear antibodies and dsDNA were negative. Hence patient was diagnosed with MMS associated with primary antiphospholipid syndrome. Antiplatelet therapy with aspirin was started instead of anticoagulation because of the risk of rupture of collateral vessels. She made a complete recovery over a period of 2 weeks with this therapy and intensive physiotherapy. The patient tolerated the aspirin without any adverse effects. A repeat MRA after 1 year, showed almost the same findings as at diagnosis though clinically she remained well with no neurological deficit.

\section{Discussion}

MMS is a progressive occlusive disease of the cerebral vasculature with particular involvement of the circle of Willis and the arteries that feed it [1]. The exact etiology of MMS is unknown; it may be multifactorial. Cases of MMS have been reported worldwide, although the incidence is higher among Asians than other ethnic groups [4]. The gender ratio (F:M) has been shown to be 1.8:1. The age distribution is bimodal. The initial peak is observed in children, typically in the first decade and the second smaller peak is in adults, typically in the fourth decade. Many disease states reported in association include Down's syndrome, Marfan syndrome, leptospirosis, tuberculous meningitis, atherosclerosis, sickle cell anemia and antiphospholipid syndrome [1, 5]. MMS had also been described in association with cigarette smoking, use of cocaine and oral contraceptives [2,6]. Characteristic presenting clinical symptoms of MMS includes headache, transient ischemic attacks, seizures hemorrhage and infarction [1].

Antiphospholipid syndrome is a heterogeneous group of autoantibodies that includes lupus anticoagulant and $\mathrm{aCL}$, which are strongly associated with arterial and venous thrombosis [3]. In patients with APS the vast majority of antibodies detected in conventional anticardiolipin assays bind to epitopes on $\beta_{2}$ GPI. The endothelial cell interaction with these antibodies has been proposed as a probable mechanism to predispose to thrombosis. Endothelial cell alterations in the vasculopathy of MMS could trigger the formation of secondary antiphospholipid antibodies responding to the exposed phospholipid components of the endothelial cell membrane [7]. On the other 


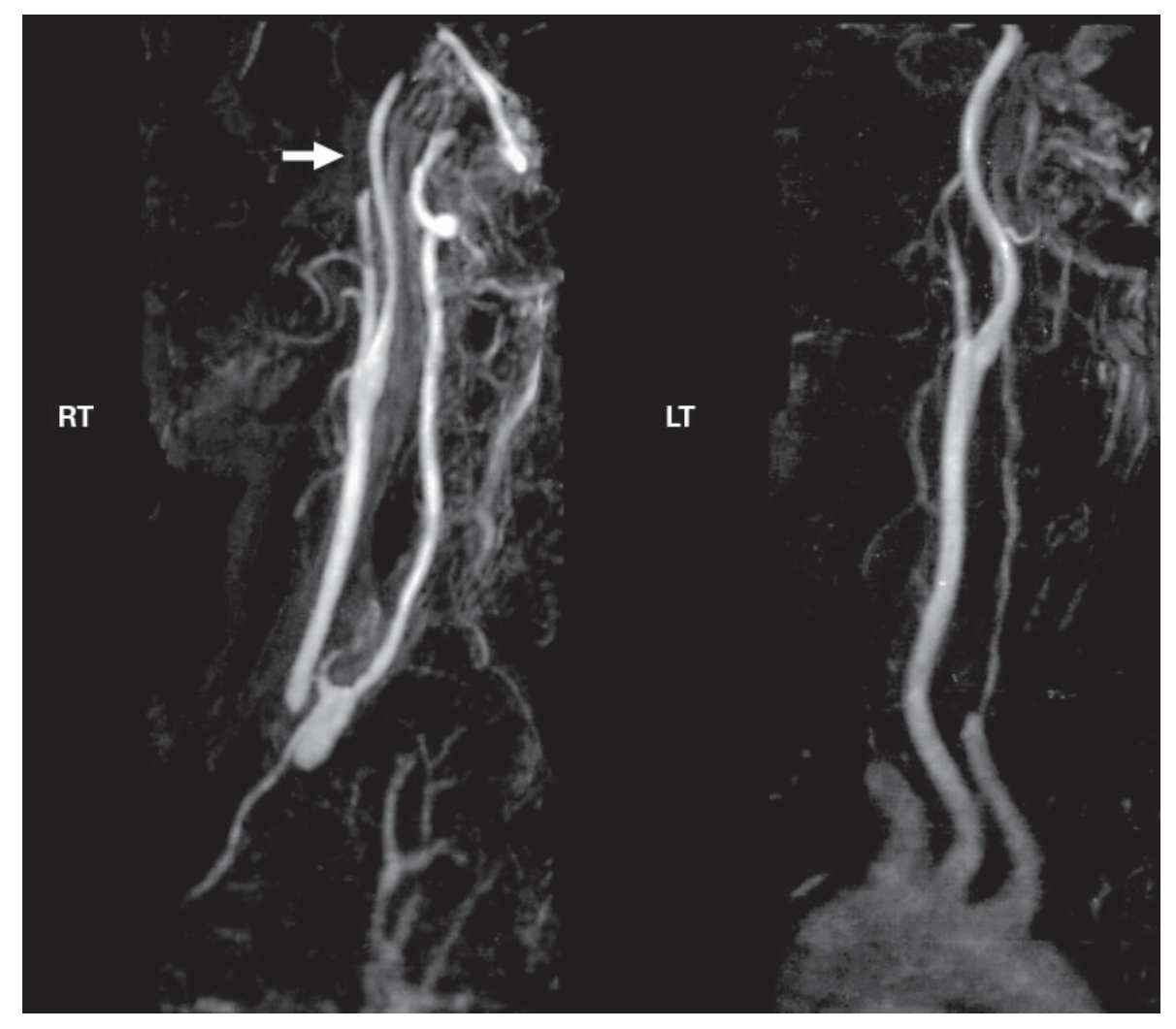

Fig. 2. Contrast-enhanced MRA of the extracranial carotids reveals tubular narrowing of the right ICA (arrow) as compared to the normal left ICA.

Fig. 3. 3D-TOF circle of Willis (coronal reformat) reveals occlusion of the supraclinoid segment of the right ICA (arrow) with narrowing of the right MCA and PCA (arrowheads).

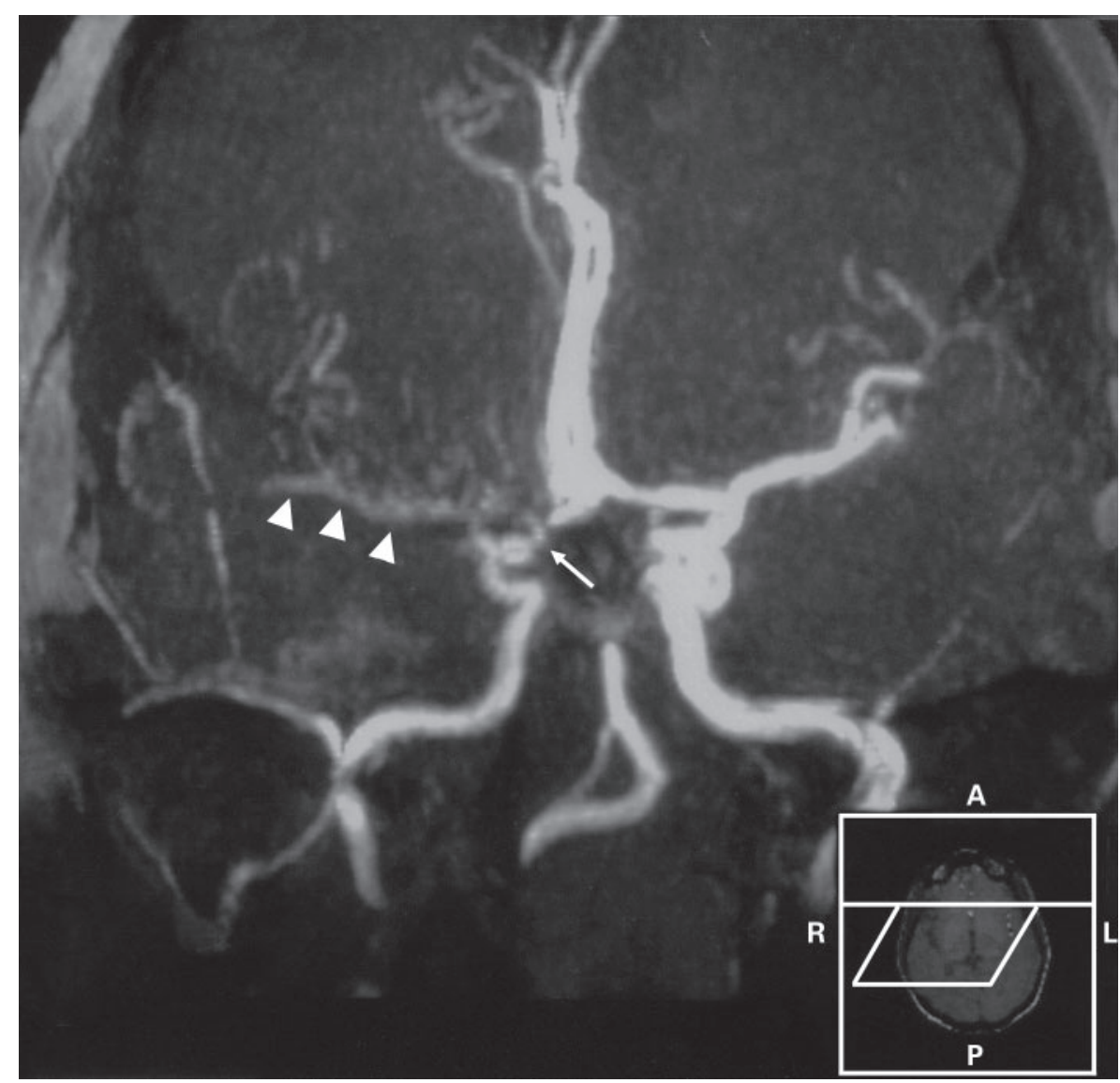


Fig. 4. 3D-TOF circle of Willis (axial reformat) shows the extensive collateralization arising from the right MCA/PCA giving the characteristic 'puff of smoke' appearance (arrowheads).

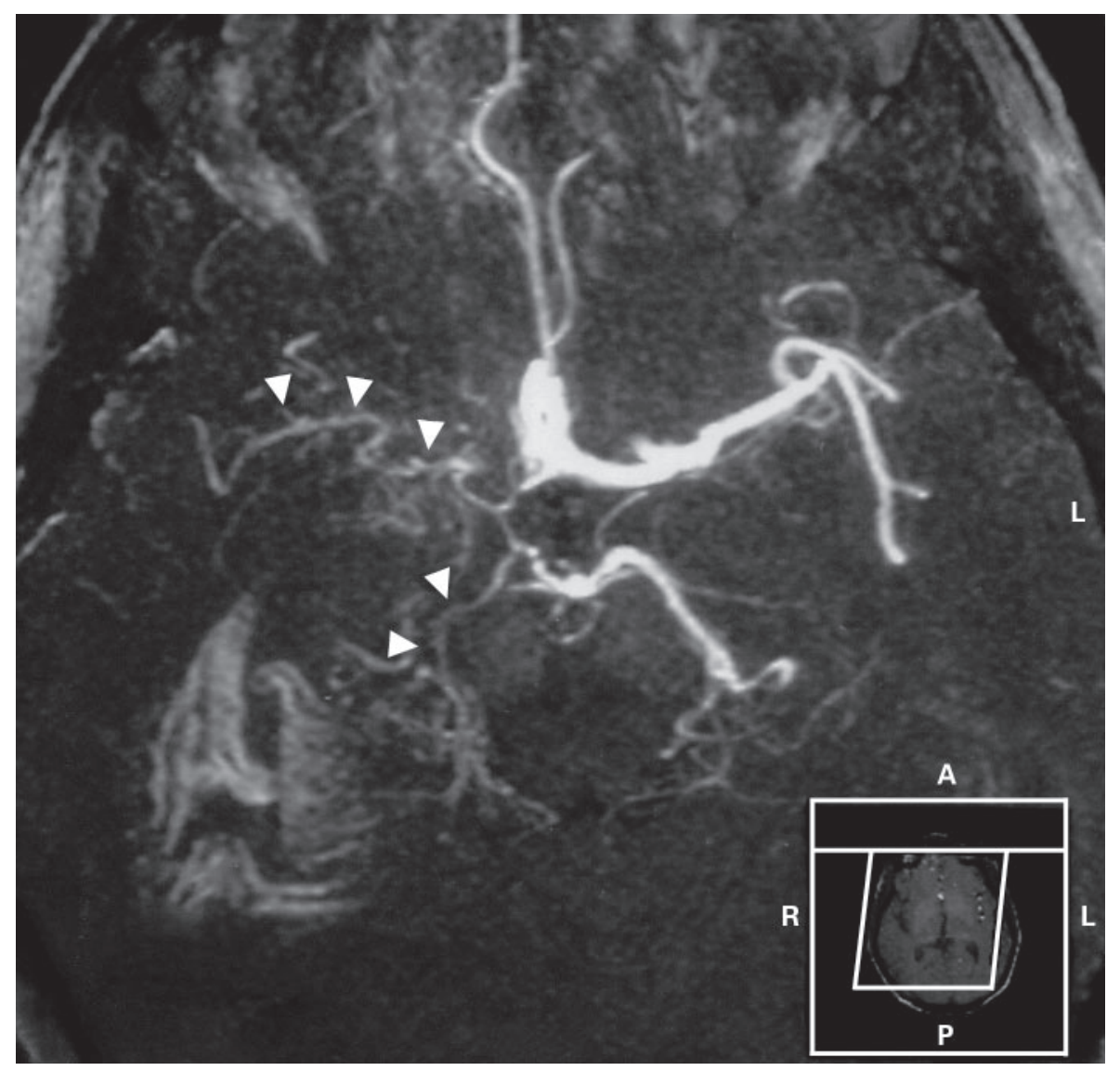

hand some authors have speculated that the development of a moyamoya type of vascular pattern in APS may be secondary to initial thrombosis and stenosis of the basal cerebral vasculature [5]. Prophylactic anticoagulant or antiplatelet therapy is usually recommended for APS patients to prevent recurrent thrombosis and progression of the vascular abnormality. However, in patients of MMS associated with APS therapeutic options are complicated by the risk of rupture of moyamoya vessels (MMVs). In view of high risk of bleeding from rupture of fragile MMVs, antiplatelet therapy was preferred in our case. During a 3-year follow-up period, our patient remained well and has shown no new symptoms of transient ischemia or stroke while on antiplatelet therapy, though the follow-up MRA remains unchanged.

\section{Conclusion}

The association of APS and moyamoya disease may present a dilemma in management. While APS is often considered to be an indication for giving anticoagulation, a trial of antiplatelet agents is preferable to anticoagulation because of the risk of bleeding from the fragile MMVs.

\section{References}

1 Suzuki J, Kodama N: Moyamoya disease - a review. Stroke 1983;14:104-109.

-2 Levine SR, Fagan SC, Pessin MS, Silbergleit R, Floberg J, Selwa JF, Vogel CM, Welch KM: Accelerated intracranial occlusive disease, oral contraceptives and cigarette use. Neurology 1991;41:1893-1901.

-3 Hughes GRV: The antiphospholipid syndrome ten years on. Lancet 1993;342:341-346.
4 Goto Y, Yene Kawa Y: Worldwide distribution of moyamoya disease. Neurol Med Chir 1992;32:883-886.

- 5 Booth F, Yanozsky R, Ross IB, Lawrence P, Oen K: Primary antiphospholipid syndrome with moyamoya-like vascular changes. Pediatr Neurosurg 1999;31:45-48.

-6 Kaufman MJ, Levin JM, Ross MH, Lange N, Rose SL, Kukes TJ, Mendelson JH, Lukas SE,
Cohen BM, Renshaw PF: Cocaine induced cerebral vasoconstriction detected in humans with magnetic resonance angiography. JAMA 1998;279:376-380.

7 Bonduel M, Hepner M, Sciuccati G, Torres AF, Tenembaum S: Prothrombotic disorders in children with moyamoya syndrome. Stroke 2001;32:1786. 